\title{
A Mobile Robot Platform for SEARCh AND RESCUE APPLICATIONS
}

\author{
Georg A. Novotny, Simon Emsenhuber, Philipp Klammer, Christoph Pöschko, \\ Florian Voglsinger \& Wilfried Kubinger
}
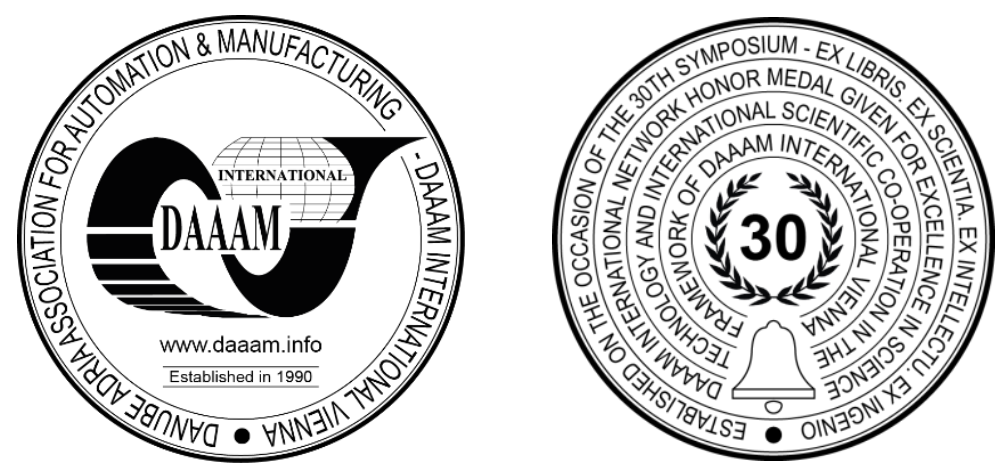

This Publication has to be referred as: Novotny, G[eorg]; Emsenhuber, S[imon]; Klammer, P[hilipp]; Poschko, C[ristoph]; Voglsinger, F[lorian] \& Kubinger, W[ilfried] (2019). A Mobile Robot Platform for Search and Rescue Applications, Proceedings of the 30th DAAAM International Symposium, pp.0945-0954, B. Katalinic (Ed.), Published by DAAAM International, ISBN 978-3-902734-22-8, ISSN 1726-9679, Vienna, Austria

DOI: $10.2507 / 30$ th.daaam.proceedings.131

\begin{abstract}
In public emergencies such as nuclear accidents or natural disasters, an immediate and accurate overview as well as an assessment of the area is the basis of all coordinated plans and actions for the rescue team. The persistent lack of such information leads to high risks and casualties for rescue workers. Mobile robots help to minimize risks and support the rescue teams with urgent information, as well as with debris clearing and search and rescue operations. This work discusses the necessities and requirements of mobile robots in search and rescue (S\&R) applications such as a nuclear disaster and evaluates an implementation based on the system architecture of the mobile S\&R robot "Robbie" of UAS Technikum Wien.
\end{abstract}

Keywords: Search and Rescue; Mobile Robot; System Evaluation; Radiation Mapping; Human Detection

\section{Introduction}

One of the main reasons why deaths follow disasters is that the rescue team takes too long to find the victims because they need to grant their own safety [1] [2]. Rescue robots have been designed for situations like these which are too dangerous for humans, e.g. hostage situations or nuclear disasters [3]. Thus S\&R robots eliminate the need of human scouts to expose themselves to hazardous environments by gaining awareness of the situation, at the disaster site, by providing immediate feedback to the rescue force prior to their entry [2]. To support developing rescue robots, a number of robotic trials and competitions have emerged since the early 2000s such as: "ELROB" - The European Land Robot Trial [4], "EnRicH" - European Robotics Hackathon [5], the "Arctic Robot Challenge" [6], "Rescue Robot League" [7], the "DARPA" - Defence Advanced Research Projects Agency [8], the "EuRoC" - European Robotics Challenges [9] or the more "EU-FP7-ICARUS" [10]project and many other. During these trials different task need to be solved ranging from $2 \mathrm{D} \& 3 \mathrm{D}$ mapping of the environment over human detection $\&$ rescue and object manipulation. 
In this paper the mechanical construction, sensor configuration and Graphical User Interface (GUI) requirements, for S\&R robots, are evaluated in the remaining part of this chapter, followed by the evaluation of the implemented hardware and developed software of the UAS Technikum Wien in section 2. The obtained results, using the reviewed implementation, are highlighted and discussed in section 3. The final section 0 summarizes this work and gives an overview concerning feature work.

\subsection{System Requirements}

The following user requirements for S\&R robots have been surveyed in [11] [12] and are summarized here for convenience in Table 1:

\begin{tabular}{|c|c|}
\hline \multicolumn{2}{|r|}{ Requirements for Search and Rescue Robots } \\
\hline Topic & Requirements \\
\hline Dimensions & $\begin{array}{l}\text { The robot platform must fit onto } 2 \text { standard euro-pallets }(120 \mathrm{~cm} \times 160 \mathrm{~cm} \mathrm{x} \\
95 \mathrm{~cm}) \text { and not weigh more than } 100 \mathrm{~kg} \text {. }\end{array}$ \\
\hline Nr. of operators & Two people must be enough to operate the S\&R robots \\
\hline Resistance & IP65 for outdoor unmanned ground vehicles (UGV) \\
\hline Autonomy & Must be possible to immediately switch from autonomous to tele-operated. \\
\hline Sensing & $\begin{array}{l}\text { Video (RGB and/or thermal) cameras for visual contact with victims, 3D } \\
\text { sensors to generate a structural map of environment }\end{array}$ \\
\hline Communication & Connection loses will occur $\rightarrow$ ad-hoc networks required \\
\hline $\begin{array}{l}\text { Command and } \\
\text { Control }\end{array}$ & Simple interfacing technologies only on high-level tasks \\
\hline Operation time & $\begin{array}{l}\text { Must be capable of working in complete darkness as well as light } \\
\text { environments. }\end{array}$ \\
\hline \multirow{3}{*}{$\begin{array}{l}\text { Graphical User } \\
\text { Interface }\end{array}$} & Camera view(s) from robot's perspective + environmental perceptions \\
\hline & Sensor and status information of initial state and sensors \\
\hline & Bird's eye view map \\
\hline
\end{tabular}

Table 1. Summary of system requirements survey, data taken from [11] [12]

\subsection{Hardware}

Disaster sites are usually covered with debris and boulders and can extend over several floors. Therefore, the base platform of the mobile robot needs to be capable of manoeuvring in rough terrain and should be suited to climb stairs. As stated in [2] track based robots are designed to operate in uneven terrains covered with debris and are therefore an ideal fit for natural disaster sites, moreover tank like tracks add stability to the whole robot system [13]. The manipulation of objects is also frequently required by $\mathrm{S} \& \mathrm{R}$ robots, whether it is for mine clearance, interaction with victims or to provide a unique camera angle [12].

Now that the system requirements have been defined, the following chapter reviews the UAS Technikum Wien's approach of integrating these requirements into one search and rescue robot.

\section{Review System Concept}

The following section reviews the requirements regarding the hardware as well as the software architecture of mobile robots to successfully participate in the ELROB and EnRicH robotic trials or other S\&R applications.

The track steered mobile robot "Tracker" of the company taurob GmbH [14] was utilized as the fundamental building stone. Thanks to the adjustable crawler tracks a high off-road mobility is provided for maximum versatility [14]. Further 
the Tracker provides two built in RGB cameras for teleoperation. Furthermore, taurob GmbH also provides a 4 degrees of freedom (DOF) robotic arm for manipulation tasks which was mounted on the base platform. The Robot Operating System (ROS) API that is provided by taurob GmbH was the final decision factor for this mobile robot base platform. The Tracker and the robotic manipulator are depicted in Fig. 1 as well as the current sensor setup and the robotic end effector (EEF).

In addition to the robot base and the manipulator a number of sensors were mounted to enable S\&R tasks. For outdoor localisation a Garmin GPS Module was attached to the sensor rig of Robbie. For GPS restricted indoor scenarios, such as the EnRicH which takes place in the nuclear power plant (NPP) Zwentendorf, depth and ranging sensors were utilized for localisation and mapping. A 3D Light Detection and Ranging (LIDAR) Velodyne PUCK VLP-16 [15] as well as two SICK TIM-551-2050001 [16] 2D LIDAR were therefore equipped, one at the front and one at the rear, on the robot. In contrary to the planar mounted 2D LIDARs the 3D LIDAR was mounted at an angle of $20^{\circ}$ tilted to the driving direction of Robbie. To further enable accurate teleoperation the LIDARs were, in combination with a corresponding software (see section 2.2), utilized to generate a $2 \mathrm{D}$ and $3 \mathrm{D}$ map of the environment thus providing the user with insights of the environment form Robbie's point of view (POV) [2] which is depicted in Fig. 3. To include the environment behind Robbie, which therefore cannot be captured by the 3D LIDAR, an additional RGB-Depth (RGB-D) camera, the Intel D435 [17], was equipped slightly facing downwards on the sensor tower of the mobile robot. On the baseplate of the EEF an additional Intel D435 was mounted to enable easier teleoperation of the EEF and image based visual servoing (IBVS) [18]. Moreover an Phidgets Spatial Inertial measurement Unit (IMU) was attached to the base platform to further improve localisation, using sensor fusion such as Extend Kalman Filters, and to provide input for a tip-over-control [19] [20] [21]. To provide an elevated POV a universal camera mount with an attached Ueye UI-3240LE [22] camera was mounted on the sensor rig. Lastly to enable radioactive and nuclear (RN) sensing the robot was equipped with a SSM1+ [23] radiometer.

The processing of these sensor data is a computationally complex process, therefore an industrial computer was also mounted on the base platform of Robbie with the following specifications:

- $1 \times$ Intel(R) Core(TM) i7-7700T (4 Cores, 8 Threads) @ 2.90GHz

- $1 \times$ GeForce GTX $1050 \mathrm{Ti}$

- $2 \times 16 \mathrm{~GB}$ DDR4 $2133 \mathrm{MHz}$

The visualisation of these different sensor readings is a difficult task. Thus an intuitive GUI was developed for Robbie which will be discussed in the next section.

\subsection{User Interface}

The user interface is the fundamental component for promoting situational awareness [12]. To emphasize this statement, the reader is made aware of the fact that a $S \& R$ robot was rejected at the 9/11 tragedy due to providing a too complex user interface [12]. Fig. 2 depictures the operator station with the appendant user interface and control panel.

As depicted in Fig. 2a) the user interface is split into two parts:

1) Log-Screen / Command Input (Figure 2a, (1))

Here all the log messages of the running software are shown, this is a necessity to detect software-system failure. In addition, a secure VPN connection to the mobile robot is established through these terminal windows in order to start/ restart any software modules. This enables a maximum level of flexibility in terms of stopping and starting applications. 


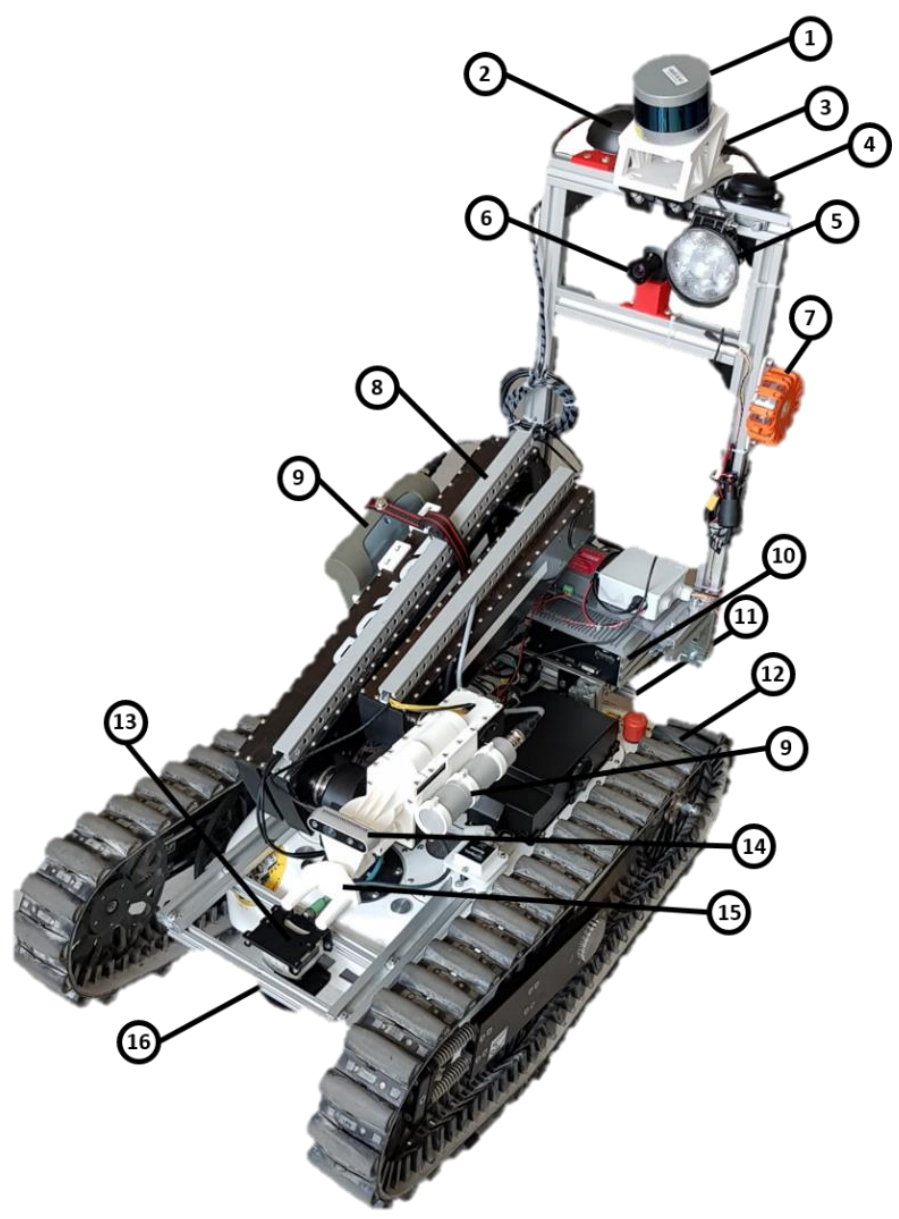

Fig. 1. Robbie hardware setup

(1) Velodyne PUCK-VLP16, (2) Wifi/ 4G Antenna, (3) Rear-Facing Intel Realsense D435, (4) Garmin GPS Module, (5) LED Headlight, (6) Ueye UI-3240LE Camera with Camera Mount, (7) Operation Indication Light, (8) Robotic Arm, (9) SSM1+ Radiatiometer with mounted Probe, (10) Embedded PC, (11) Rear-Facing Internal Camera and SICK TIM551-2050001, (12) taurob Tracker, (13) Front-facing TIM-551-2050001, (14) Intel Realsense D435 mounted on EEF,

(15) EEF, (16) Front-Facing Internal Camera and Internal Headlights

\section{2) GUI (Figure $2 \mathrm{a},(2))$}

The second part of the user interface, the GUI, is utilized to allow the operator to perceive the environment of Robbie's POV which is a necessity for S\&R robots [12]. This is achieved by live streams of the cameras on the right side of the GUI (see Fig. 2b, (8)). The standard configuration streams the internal front and rear facing cameras of the Tracker as well the elevated RGB camera for a higher POV. Further, the 2D map, created by the SLAM approach discussed in section 2.2, is visualised in the middle part of the GUI as visualised in Fig. 2b) (7), therefore creating a bird's eye view for the operator. Lastly sensor readings (Fig. 2b) (4)), such as the inside temperature, the battery voltage and the estimate time till shutdown as well as the detected ionizing radiation in Counts per Second (cps). Thus the developed GUI visualizes all suggestions of a good user interface evaluated in [12], which further enables the optimization and interoperability of the available resources and accelerates access to the victims [11].

Supplementary it is also possible to visualise additional sensor readings and information by a simple mouse click (Fig. 2b) (5)), such as a 3D map, the additional camera at the gripper and the rear facing camera, the autonomous detected humans, see Fig. 2c) or the local and global costmap for the autonomous drive. Further the GUI can also be used for teleoperation of the robotic arm in case the connection to the control panel fails, therefore delivering a redundant teleoperation system (Fig. 2c (6)). 
a)

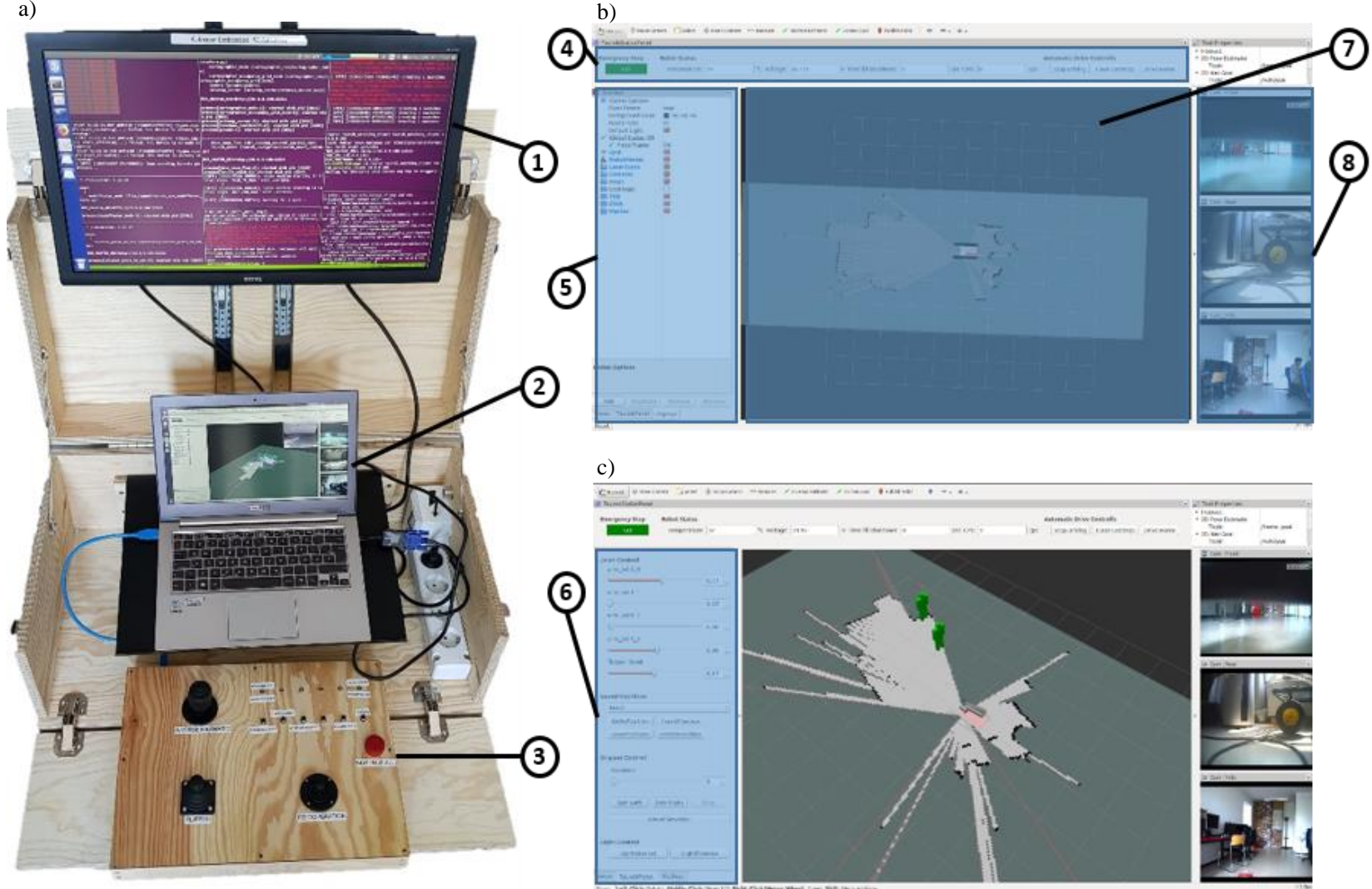

Fig. 2. a) Complete operator station b) GUI c) GUI

(1) Logscreen / Command input, (2) GUI depicted in more detail in b) and c), (3) Control panel for teleoperation, (4) Sensor readings and emergency of switch, (5) Topic visualisation checkbox, (6) Additional teleoperation toolbox, (7) Map visualisation toolbox, (8) Image stream from Robbie's POV

The control panel for teleoperation (Fig. 2 (3)) is used to operator Robbie. Here the steering of the base platform as well as the robot-arm is handled.

\subsection{Software}

To evaluate the previously introduced sensors and to steer the actuators the Robot Operating System (ROS) is used as high-level API. Besides the basic communication with the actuators, sensors and the operator station ROS is used because of its scalability and open source infrastructure [24]. Thus with ROS in combination with the taurob Tracker a basic teleoperated mobile robot is provided to build on top. Therefore, making it a perfect fit for R\&D applications. ROS further provides a robot visualisation toolkit called "rviz" [25]. To further improve the tele-operation process a graphical user interface plugin for rviz, which displays all sensor data and allows for easier teleoperation of the robot arm, was developed using qt, as seen in Fig. 2. For 2D respectively 3D map generation the open-source frameworks Cartographer [26] and Octomap [27] where implemented. The main benefit of the Cartographer algorithm being its capability to detect and calculate online loop closure with graph optimisation therefore minimizing the absolute translational and rotational errors when generating a map. Cartographer matches each consecutive scan against a small submap and uses nonlinear optimisation to calculate the loop closure [26]. Octomap, on the other hand, uses probabilistic occupancy estimation of the 3D space and represents the environment in octrees which consists of occupied voxels [27]. Fig. 3a) and Fig. 3c) visualize a generated 2D respectively 3D map using these SLAM approaches utilizing the LIDAR sensors listed in section 1.2 taken during the EnRicH 2019 trial.

To overcame the need of manual victim detection and mapping the UAS Technikum Wien team developed a ROS package that builds on top of Octomap and YOLO-ROS [28], a convolutional neural network (CNN) for object detection in RGB images. It utilizes the ray-casting functionality of Octomap and the bounding box provided by YOLO-ROS, given the 6 DOF transformation between the map frame and the RGB camera frame, to compute the $\mathrm{x}$ and $\mathrm{y}$ coordinates of the 
victim. The detected victim is then visualized on the $2 \mathrm{D}$ map for the operator (see Fig. 2c)). The fact that this approach uses 2 different sensors to calculate the position of the victim is justified by that fact that this way even thermal cameras can be used for victim detection. This would enable the robot to be functional in various environmental circumstances such as fog, smoke, rain, and snow

Further an automatic drive ROS package was developed utilizing the move-base-flex framework [29], a new flexible navigation framework for the navigation stack in ROS, and SMACH [30], a task-level architecture to build complex robot behaviours using state machines. Currently two path-planners are implemented in this framework. First the Timed Elastic Band (TEB)-planner is called. It is an elastic band planner, which considers the travel time. The movement is not calculated via the simulated forces within the virtual elastic band, but via optimization of the travel time and distance [31]. The planner calculates several viable paths and chooses the fastest one. Should the planner not reach the goal the recovery behaviours are called. After each behaviour call the planner tries the reach the goal again. Should he still not be able to reach the goal, the next recovery behaviour is called. After the three implemented behaviours have been executed the local planner is switched to the Dynamic Window Approach (DWA) - algorithm. It breaks the global plan into smaller windows. Only the current and the next window are used to calculate the path [32]. The velocity within the next window are calculated via the current robot velocity, the possible acceleration of the robot and objects that must be avoided. The goal tolerance of the DWA planner is increased to ensure that the goal position is reachable. Should the planner not be able to reach the given goal, the three recovery behaviours are called in the same way as with the TEB-planner. Should the system after the call of all recovery behaviours still not be able to reach the goal the execution of the local planner is cancelled. The SMACH-script returns an error and waits for a new goal. The first implemented recovery behaviour clears the cost maps, the second moves the robot back for $0.3 \mathrm{~m}$ or 5 seconds and the third rotates the robot $360^{\circ}$ on the spot.

During the exploration task the radioactivity is measured, in Counts per Seconds, continuously with the SSM1+ radiometer. After the exploration a Gaussian Process is used to estimate the nuclear radiation for the surrounding area of the robot's path. With OpenCV the amount of radiation is visualized and overlaid over the 2D map, together with a legend for the map. Via coloured markers the radioactivity is also visualized in the 3D map in rviz.

To further assist the tele-operated manipulation tasks an inverse kinematic was implemented for the robotic arm. Based on the "Blind Search" algorithm [33], a fast and reliable solution for kinematics with more than three degrees of freedom, the program processes movement orders and moves the TCP of the EEF accordingly by calculating the necessary angles for the joints and sending the respective commands to the motors. The TCP moves along a straight line from starting to finishing point, as this motion is best suited for the manipulation task at EnRicH.

The following section 3 now introduces the results achieved using this system concept.

\section{Results and Discussion}

The following section evaluates the System and Technology Readiness Level (SRL) (TRL) of Robbie followed by examples of created maps using the current sensor configuration.

Readiness Level

\begin{tabular}{|c|c|c|c|}
\hline \multicolumn{4}{|c|}{ System Readiness Level } \\
\hline Topic & Requirements & Robbie & \\
\hline Dimensions & $\begin{array}{l}\text { The robot platform should fit onto } 2 \text { standard euro-pallets } \\
(120 \mathrm{~cm} \times 160 \mathrm{~cm} \times 95 \mathrm{~cm}) \text { and not weigh more than } 100 \mathrm{~kg} \text {. }\end{array}$ & $112 \times 58 \times 120 \mathrm{~cm}$, ca. $75 \mathrm{~kg}$ & \\
\hline Nr. of Operators & Two people must be enough to operate the $S \& R$ robots & Only one operator required & $\nabla$ \\
\hline Resistance & IP65 for outdoor unmanned ground vehicles (UGV) & $\begin{array}{l}\text { Currently no IP certificate due to } \\
\text { active cooling }\end{array}$ & 区 \\
\hline Autonomy & $\begin{array}{l}\text { Must be possible to immediately switch from automation to } \\
\text { tele-operated. }\end{array}$ & $\begin{array}{l}\text { Can be easily switched of using the } \\
\text { GUI or control panel }\end{array}$ & $\nabla$ \\
\hline
\end{tabular}




\begin{tabular}{|c|c|c|c|}
\hline Sensing & $\begin{array}{c}\text { Video (RGB and/or thermal) cameras for visual contact } \\
\text { with victims, 3D sensors to generate a structural map of } \\
\text { environment }\end{array}$ & $\begin{array}{l}\text { Five image-streams provided as } \\
\text { well as } 2 \mathrm{D} \text { and } 3 \mathrm{D} \text { maps }\end{array}$ & $\nabla$ \\
\hline Communication & Connection loses will occur $\rightarrow$ ad-hoc networks required & $\begin{array}{l}\text { Automatic drive counters } \\
\text { connectivity problems }\end{array}$ & $\nabla$ \\
\hline $\begin{array}{l}\text { Command and } \\
\text { Control }\end{array}$ & Simple interfacing technologies only on high-level tasks & $\begin{array}{c}\text { Intuitive GUI and operator station } \\
\text { developed }\end{array}$ & $\nabla$ \\
\hline Operation Time & $\begin{array}{l}\text { Must be capable of working in complete darkness as well } \\
\text { as light environments. }\end{array}$ & $\begin{array}{l}\text { Can be operated during day and } \\
\text { night due to two LED headlights }\end{array}$ & $\square$ \\
\hline $\begin{array}{c}\text { Energy } \\
\text { Requirements }\end{array}$ & $\begin{array}{c}\text { Energy consumption should be lower than 2kVA for } \\
\text { recharging }\end{array}$ & $0.36 \mathrm{kVA}$ & $\nabla$ \\
\hline \multirow{3}{*}{$\begin{array}{l}\text { Graphical User } \\
\text { Interface }\end{array}$} & $\begin{array}{l}\text { Camera view(s) from robot's perspective + environmental } \\
\text { perceptions }\end{array}$ & $\begin{array}{l}\text { Three image-streams on start-up, } \\
\text { additional } 2 \text { can be started manually }\end{array}$ & $\nabla$ \\
\hline & Sensor and status information of initial state and sensors & $\begin{array}{l}\text { Battery Voltage, Remaining } \\
\text { operation time and sensor readings } \\
\text { in status-bar }\end{array}$ & $\nabla$ \\
\hline & Bird's eye view map & $\begin{array}{l}\text { 2D map on start-up, 3D map can be } \\
\text { visualised manually }\end{array}$ & $\nabla$ \\
\hline
\end{tabular}

Table 2. Evaluation of Robbie's applicability using the survey in [11]

Using the survey results of [11] Robbie's SRL is defined as 9/10.

\begin{tabular}{|c|c|}
\hline \multicolumn{2}{|c|}{ Technology Readiness Level } \\
\hline Hardware & Software \\
\hline $\begin{array}{l}\text { Tracker: Problem with overheating } \\
\text { Sensor Rig: Tower is distorting }\end{array}$ & $\begin{array}{l}\text { Base Framework: ROS } 1 \text { therefore no real-time support } \\
\text { 2D SLAM: Problem with recursive trajectory optimisation on } \\
\text { loop-closure } \\
\text { YOLO-ROS: Not completely stable }\end{array}$ \\
\hline
\end{tabular}

Table 3. Evaluation of Robbie's TRL

Using

Table 3. Robbie's TRL is defined as follows: Hardware is $6 / 8$ and Software is $4 / 6$.

\section{Created Maps}

The following Fig. 3 visualises the same environment, captured during the EnRicH 2019 trial, as a plain 2D, 2D with marked radioactive sources and the corresponding 3D map. As can be clearly seen in fig. 3a) the detected loop closure by the cartographer node distorted the map resulting in crooked walls. This loop-closure also lead to the fact that two radioactive sources were merged to one by our radioactive mapping approach since the odometry, of the mobile robot, was not recursively recalculated on loop-closure. 

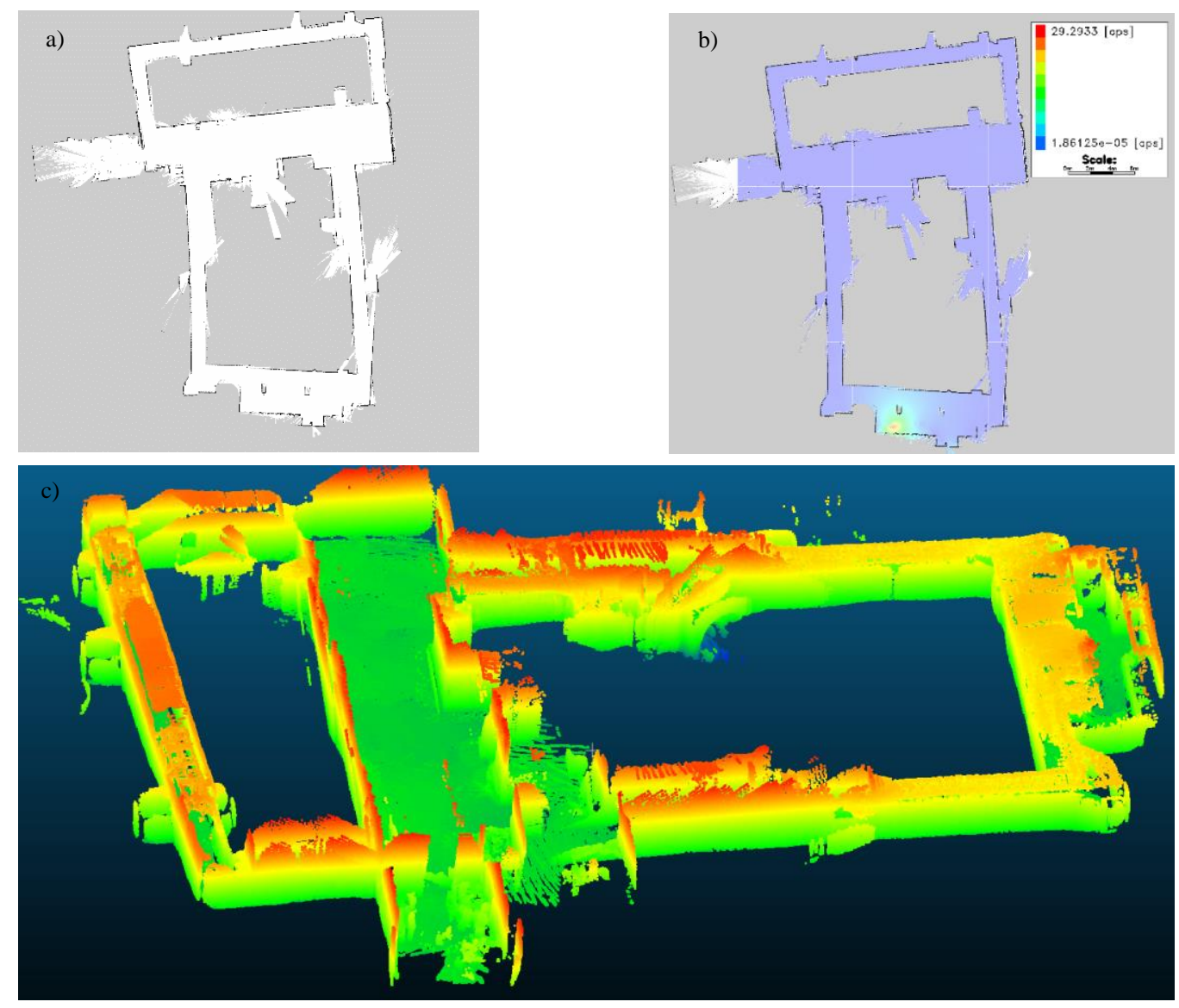

Fig. 3. a) generated 2D map, b) 2D map overlaid with radioactivity measurements, c) generated 3D map

As seen in fig. 3c) the generated 3D map is only partly dense, which means that not in all regions a dense 3D reconstruction was possible. This may be due to the fact that the 3D depth sensors have a too small range or that the mobile robot was driven too fast through these regions. To further improve the density of the 3D Map as well as the teleoperation task of the EEF, the attached RGB-D camera could be exchanged with a stereo camera, for higher resolution, using an adapted version of the algorithms presented in [34].

\section{Conclusion and Outlook}

In this paper the necessities of search and rescue robots were investigated in terms of system requirements from the operator's point of view, further the UAS Technikum Wien's approach of implementing the requirements was reviewed. Search and rescue robots must cover a wide range of application areas. Starting with the teleoperation of the robot via autonomous object detection and mapping up to the processing and visualisation of the sensor data for the operator. The search and rescue robot of the UAS Technikum Wien is capable of generating various Maps (2D and 3D), has autonomous capabilities such as human victim detection or the autonomous drive and has an easy to use graphical user interface for the operator. The Tracker base platform in combination with the robotic arm and end effector enable high off-road mobility and provide maximum versatility for manipulation. It's only downside being the currently not stable human detection and the problem with the radioactive mapping on loop-closure of the Cartographer algorithm.

Further projects will tackle the tasks of teleoperation of the robotics arm using motion controllers, upgrading the human detection package by fusing the already existent RGB data with pointcloud data using bayesian sensor fusion and visual servoing with reinforcement learning for optimal gripper positioning.

\section{Acknowledgments}

This project has been partly funded by MA23 - City of Vienna within the Project Call 16-02 "Photonics: Foundations and industrial applications" and by the Austrian Ministry of Defense R\&D contract "Autonomous Land Systems". 


\section{References}

[1] Garyson, S. (2014). Search \& Rescue using Multi-Robot Systems, Available from: https://www.semanticscholar.org/paper/Search-\%26-Rescue-using-Multi-Robot-SystemsGrayson/732decce6717f005682a799b8a0f3d91cfc494d5 Accessed: 2019-07-01

[2] Kostavelis, M., N. \& Gasteratos, A. (2017). Robots in Crisis Management: A Survey, In: in Information Systems for Crisis Response and Management in Mediterranean Countries, Dokas, M., I.; et al. (Ed.), pp. 43-56, Springer, 978- 3-319-67633-3, Xanthi, Greece

[3] Kiyani, M., N. \& Khan, M., U., M., (2016). A prototype of search and rescue robot, 2016 2nd International Conference on Robotics and Artificial Intelligence (ICRAI), Rawalpindi, Pakistan, ISBN 978-1-5090-4059-9, pp. 208-213, IEEE, DOI: 10.1109/ICRAI.2016.7791255

[4] ELROB - The European Land Robot Trial, "ELROB - The European Land Robot Trial," [Website]. Available from: https://www.elrob.org Accessed: 2019-07-15

[5] EnRicH - The European Robotics Hackathon, "ENRICH - The European Robotics Hackathon," [Website]. Available from: https://www.enrich.european-robotics.eu/ Accessed: 2019-07-15

[6] ARCTIC ROBOT CHALLENGE - ROBOTICS IN THE ROUGH, "ARCTIC ROBOT CHALLENGE ROBOTICS IN THE ROUGH," [Website]. Available: https://arcticrobotchallenge.com/ Accessed: 2019-07-15

[7] Rescue Robot League | RoboCup German Open, "Rescue Robot League | RoboCup German Open 2019," [Website]. Available from: https://www.robocupgermanopen.de/de/major/rescue Accessed: 2019-07-15

[8] DARPA - Defense Advanced Research Projects Agency, "Defense Advanced Research Projects Agency," [Website]. Available from: https://www.darpa.mil/ Accessed: 2019-07-15

[9] EUROC - European Robotics Challenges, "EUROC - European Robotics Challenges," [Website]. Available from: http://www.euroc-project.eu Accessed: 2019-07-15

[10] FP7-ICARUS, "FP7-ICARUS," [Website]. Available from: http://fp7-icarus.eu/ Accessed: 2019-07-15

[11] Doroftei, D.; Matos, A. \& De Cubber, G. (2014). Designing Search and Rescue Robots towards Realistic User Requirements, Available from: https://www.semanticscholar.org/paper/Designing-Search-and-RescueRobotstowards-User-Doroftei-Matos/164f9dfc99e12bfe1f8b25d484fbf1fe023fe404 Accessed: 2019-07-10

[12] Murphy, R., R.; Tadokoro, S. \& Kleiner, A. (2016). Disaster Robotics, In: Springer Handbook of Robotics, Siciliano, B. (Ed.) \& Khatib, O. (Ed.), pp. 1577-1604, Springer, ISBN 978-3-319-32552-1, DOI: 10.1007/978-3-319-325521_60

[13] Oliveria, J.; et al. (2018). A Review on Locomotion Systems for RoboCup Rescue League Robots, In: RoboCup 2017: Robot World Cup XXI, Akiyama, H.; et al. pp. 265-276, Springer, ISBN 978-3-030-00308-1, DOI: 10.1007/978-3-030-00308-1_22

[14] Taurob GmbH, „taurob“, taurob GmbH, [Website]. Available from: http://www.taurob.com Accessed: 2019-07-17

[15] Velodyne, „Puck ${ }^{\mathrm{TM}}$ - Velodyne Lidar“, Velodyne, [Website]. Available from: https://velodynelidar.com/vlp-16.html Accessed: 2019-07-17

[16] SICK, „TIM551-2050001 | Mess- und Detektionslösungen | SICK,“ SICK, [Website]. Available from: https://www.sick.com/at/de/mess-und-detektionsloesungen/2d-lidar-sensoren/tim5xx/tim551-2050001/p/p343045 Accessed: 2019-07-17

[17] Intel, „Depth Camera D435 - Intel® RealSense ${ }^{\text {TM }}$ Depth and Tracking Cameras“, Intel, [Website]. Available from: https://www.intelrealsense.com/depth-camera-d435/ Accessed: 2019-07-17

[18] Chaumette, F.; Hutchinson, S. \& Corke, P. (2016). Visual Servoing and Visual Tracking, In: Springer Handbook of Robotics, Siciliano, B. (Ed.) \& Khatib, O. (Ed.), pp. 563-583, Springer, ISBN 978-3-540-30301-5, DOI: 10.1007/978-3-540-30301-5_25

[19] Choi, B. \& Lee, Y. (2018). Practical control of a rescue robot while manoeuvring on uneven terrain, Journal of Mechanical Science and Technology Vol. 16, No. 5, 2018, pp. 2021-2028, ISSN 1976-3824, DOI: 10.1007/s12206018-0410-7

[20] Khoshelham, K. \& Zlatanova, S. (2016). Sensors for Indoor Mapping and Navigation, Sensors 2016, Vol. 16, DOI: https://doi.org/10.3390/s16050655

[21] Kumar, G., A; et al. (2017). A LiDAR and IMU Integrated Indoor Navigation System for UAVs and Its Application in Real-Time Pipeline Classification, Sensors 2017, Vol. 17, DOI: 10.3390/s17061268

[22] Imaging-Development-System-GmbH, „UI-32401e“, IDS, [Website]. Available from: https://de.idsimaging.com/store/ui-3240le.html Accessed: 2019-07-17

[23] Seibersdorf-Laboratories, „Messgerät SSM1+ - SEIBERSDORF LABORATORIES,“ Seibersdorf Laboratories, [Website]. Available from: https://www.seibersdorf-laboratories.at/produkte/ionisierendestrahlung/messgeraeteanlagen/messgeraet-ssm1 Accessed: 2019-07-17

[24] Hellmund, A.; et al. (2016). Robot operating system: A modular software framework for automated driving, In: 2016 IEEE 19th International Conference on Intelligent Transportation Systems (ITSC), pp. 1564-1570, IEEE, ISSN 2153-0017, DOI: 10.1109/ITSC.2016.7795766 
[25] Kam, H., R.; et al. (2015). RViz: a toolkit for real domain data visualization, In: Telecommunication Systems, Vol 60, No. 2, pp. 337-345, Springer, ISSN 1572-9451, DOI: 10.1007/s11235-015-0034-5

[26] Hess, W. et al. (2016). Real-time loop closure in 2D LIDAR SLAM, In: 2016 IEEE International Conference on Robotics and Automation (ICRA), Stockholm, 2016, pp. 1271-1278, IEEE, ISBN 978-1-4673-8026-3 DOI: 10.1109/ICRA.2016.7487258

[27] Hornung, A.; et al. (2013). OctoMap: An Efficient Probabilistic 3D Mapping Framework Based on Octrees, In: Autonomous Robots Vol. 34, No. 3, pp. 189-206, Springer, ISSN 1573-7527, DOI: https://doi.org/10.1007/s10514012-9321-0

[28] Bjelonic, M. (2016-2018). Real-Time Object Detection for ROS. [Website]. Available from: https://github.com/leggedrobotics/darknet_ros Accessed: 2019-07-18

[29] Pütz, S.; Simón, J., S. \& Hertzberg, J. (2018). Move Base Flex: A Highly Flexible Navigation Framework for Mobile Robots. 2018 IEEE/RSJ International Conference on Intelligent Robots and Systems (IROS), pp. 3416-3421, IEEE, ISSN 2153-0866, DOI: 10.1109/IROS.2018.8593829, Madrid, Spain

[30] Bohren, J. \& Cousins, S. (2010). The SMACH High-Level Executive [ROS News]. In: IEEE Robotics Automation Magazine Vol 17. No 12, pp. 18-20, DOI: 10.1109/MRA.2010.938836, IEEE

[31] Roesmann, C.; et al. (2012). Trajectory modification considering dynamic constraints of autonomous robots. 2012 7th German Conference on Robotics (ROBOTIK), pp. 1-6. IEEE, ISSN 978-3-8007-3418-4, Berlin, Germany

[32] Fox, D.; Burgard, W. \& Thrun, S. (1997). The dynamic window approach to collision avoidance. In: IEEE Robotics Automation Magazine Vol 4. No 1, pp 23-33, DOI: 10.1109/100.580977, IEEE

[33] Cubero, S. (2008). Blind Search Inverse Kinematics for Controlling All Types of Serial-link Robot Arms. In: Billingsley J., Bradbeer R. (Ed.) Mechatronics and Machine Vision in Practice. Springer, Berlin, Heidelberg

[34] Marko, T., \& Kubinger, W. (2018). Automatic Stereo Camera Calibration in Real-World Environments Without Defined Calibration Objects. Proceedings of the 29th DAAAM International Symposium, pp. 0102 - 0108 , B. Katalinic (Ed.), Published by DAAAM International, ISBN 978-3-902734-20-4, ISSN 1726-9679, Vienna, Austria DOI: $10.2507 / 29$ th.daaam.proceedings.014 